\title{
Single-strand DNA Binding of Actinomycin D with a Chromophore 2-Amino to 2-Hydroxyl Substitution
}

\author{
Hoon Yoo* and Randolph L. Rill ${ }^{\dagger}$ \\ Department of Pharmacology and Dental Therapeutics, College of Dentistry, Chosun University, Gwangju 501-759, Korea \\ 'Department of Biomedical Sciences, The Florida State University, Tallahassee, FL 32306-4300, USA
}

Received 15 November 2002, Accepted 24 January 2003

\begin{abstract}
A modified actinomycin D was prepared with a hydroxyl group that replaced the amino group at the chromophore 2-position, a substitution known to strongly reduce affinity for double-stranded DNA. Interactions of the modified drug on single-stranded DNAs of the defined sequence were investigated. Competition assays showed that 2hydroxyactinomycin D has low affinity for two oligonucleotides that have high affinities $\left(K_{\mathrm{a}}=5-10 \times 10^{6}\right.$ $\mathbf{M}^{-1}$ oligomer) for 7-aminoactinomycin $\mathrm{D}$ and actinomycin D. Primer extension inhibition assays performed on several single-stranded DNA templates totaling around $1000 \mathrm{nt}$ in length detected a single high affinity site for 2hydroxyactinomycin $\mathrm{D}$, while many high affinity binding sites of unmodified actinomycin D were found on the same templates. The sequence selectivity of 2hydroxyactinomycin $D$ binding is unusually high and approximates the selectivity of restriction endonucleases. Binding appears to require a complex structure, including residues well removed from the polymerase pause site.
\end{abstract}

Keywords: Actinomycin D, DNA, Replication, Retroviruses, Transcription

\section{Introduction}

Actinomycin D (ActD) is a well-known antibiotic and antitumor agent, which binds tightly to double-stranded DNA (dsDNA) and inhibits transcription (Goldberg and Friedman, 1971; Waring, 1981; Phillips and Crothers, 1986). For a long time, it was thought that ActD binds only to dsDNA, but high affinities for selected sites in single strands were recently demonstrated, suggesting that transcription inhibition by ActD

*To whom correspondence should be addressed.

Tel: 82-62-230-6894; Fax: 82-62-230-6894

E-mail: hoon_yoo@chosun.ac.kr could involve binding to single-stranded DNA (ssDNA) intermediates (Sobell, 1985; Wadkins and Jovin, 1991; Rill and Hecker, 1996; Wadkins et al., 1996; Wadkins et al., 1998). These findings also suggest that drugs that are targeted to ssDNA might be developed using actinomycins as lead compounds. In fact, ActD strongly inhibits (-) strand transfer by HIV reverse transcriptase (Mitsuya et al., 1990; WongStaal et al., 1992; Davis et al., 1998; Guo et al., 1998; Jeeninga et al., 1998), viral ligase (Shuman, 1995), and viral helicase (Tuteja, 1997); hence, these drugs could have antiretroviral activities by binding to ssDNA replication intermediates.

Hundreds of ActD derivatives have been reported (Brockmann, 1974; Mauger, 1991; Takusagawa, 1996; Tong, 1996; Takusagawa, 1997). Several biologically-active forms bind strongly to double-stranded DNA. The ActD chromophore (Fig. 1) intercalates into dsDNA with two cyclic pentapeptide chains in the minor groove. A strong preference for intercalation into 5'-GpC steps is explained by two hydrogen bonds that are formed to each 5'-guanine (Sobell and Jain, 1972; Crothers and Müller, 1974; Krugh and Chen,

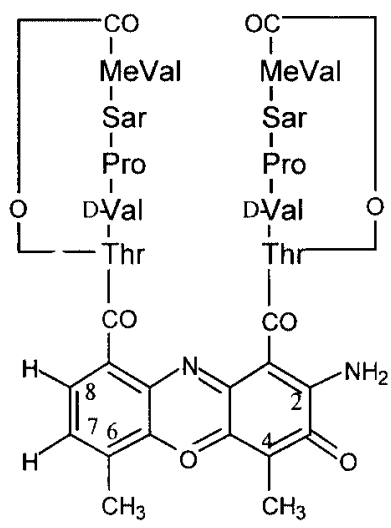

Actinomycin D

Fig. 1. The actinomycin D chromophore structure. 
1975; Jones et al., 1988; Zhou et al., 1989; Kamitori and Takusagawa, 1992). Takusagawa has described ActD derivatives that bind RNA:DNA hybrids (Shinomiya et al., 1995; Takusagawa, 1997). Except for the 7-amino derivative, virtually nothing is known about the binding of modified actinomycins to single-stranded DNA.

Since several contacts are responsible for the high affinity of ActD for dsDNA, and flexible ssDNA can alter the conformation to recognize ligands with appropriate interaction sites, then ActD derivatives with low dsDNA affinity are candidates for drugs that are selective for single-stranded DNA. The seemingly slightly-modified 2hydroxyactinomycin D was found to have little general affinity for either single- or double-stranded DNA, but a high affinity site was located within the lac $Z$ gene. Further studies with a model oligonucleotide indicated that 2-hydroxyActD binds to a complex secondary structure.

\section{Materials and methods}

Materials Actinomycin D and 7-aminoActD were from Sigma Chemical Co. (St. Louis, USA) and used without further purification. Drug concentrations were determined using published extinction coefficients (ActD, 24,500 $\mathrm{M}^{-1} \mathrm{~cm}^{-1}$ at $440 \mathrm{~nm}$ (Crothers and Müller, 1974); 7-aminoActD, $21900 \mathrm{M}^{-1} \mathrm{~cm}^{-1}$ at $505 \mathrm{~nm}$ (Sengupta and Schaer, 1978), and 2-hydroxyActD, $18500 \mathrm{M}^{-1} \mathrm{~cm}^{-1}$ (Moore et al., 1975)). Exonuclease, Sequenase ${ }^{\circledR}$, and restriction endonuclease TfiI were from United States Biochemical Corp. (USB, Cleveland, USA). Electrophoresis reagents were obtained from Fisher Scientific (Pittsburgh, USA).

Preparation of 2-hydroxyAct D Actinomycin D was converted to 2-hydroxyactinomycin $\mathrm{D}$, as described by Meienhofer (Moore $e t$ al. 1975) by heating in $10 \% \mathrm{HCl}$ at $60^{\circ} \mathrm{C}$. The product was purified by chromatography (Beckman Instruments, Fullerton, USA) on a reverse phase column $\left(1 \times 25 \mathrm{~cm}, \mathrm{C}_{18}\right.$ YMC-Pack ODS $)$ that was eluted with a linear gradient of $50 \%$ to $70 \%$ acetonitrile with detection at $280 \mathrm{~nm}$. Formation of 2-hydroxyActD was monitored in preliminary experiments by HPLC of reaction aliquots that were taken at different times. The conversion of ActD to 2-hydroxyActD was progressive and complete within $5 \mathrm{~h}$.

The 2-hydroxyActD peak was unexpectedly broad. Spectra of several sections of the peak were taken using the scanning option of the Beckman Diode Array 168 detector, since the actinomycin spectrum is sensitive to chromophore alterations. The spectrum did not change across the band, a result that was consistent with different conformations of 2-hydroxyActD, and not with different ring-altered components.

The spectrum of 2-hydroxyActD will not change if a peptide ring is broken, but the retention times of ring-opened forms should depend on $\mathrm{pH}$ because the terminal carboxyl group will be protonated at low $\mathrm{pH}$. No differences in peak shape or elution time were noted when chromatography was performed at neutral $\mathrm{pH}$ or in a solution that was acidified with $0.1 \%$ trifluoroactic acid, indicating that peptide hydrolysis did not occur. Rechromatography of several fractions taken across the 2-
hydroxyActD peak yielded exactly the same broad peak shape and retention time as the original sample. This provides direct evidence that the peak breadth was due to slow equilibration between the conformers.

Primer extension assays Two plasmids that were used for primer extension assays, pKHQ-(+)1 and pKHQ-(-)2, were described previously (Rill and Hecker, 1996; Hecker and Rill, 1997). The plasmids have short target inserts that together contain all possible base quartets in a minimum length sequence. The inserts are free of long self-complementary regions. Therefore, they have little possibility of a local secondary structure. Assays were performed as described on the four single-stranded target sequences from the two plasmids that were distinguished as (+) $1 \mathrm{R}$ and $(+) 1 \mathrm{~F}$ and (-)2R and (-)2F, where R and F indicate accessibility for the M13 Reverse (R) or Forward (F) primers (Rill and Hecker, 1996; Hecker and Rill, 1997). Additional assays were performed using phage M13mp18 circular ssDNA from USB as a template (Yanisch-Perron, et al., 1985).

Preparation and purification of oligonucleotides The oligonucleotides were synthesized on an Applied Biosystems Model 391A DNA synthesizer, deblocked with concentrated ammonium hydroxide, and purified using a Pharmacia Mono Q column and Pharmacia FPLC system. The oligomers were recovered and desalted using SEP-PAK $\mathrm{C}_{18}$ cartridges (Waters Associates, Milford, USA). The oligonucleotide concentrations were determined from $\mathrm{A}_{260 \mathrm{~nm}}$ (Cantor, 1970). An 18-mer oligonucleotide was 5 '-end labeled with ${ }^{32} \mathrm{P}$ - $[\alpha]$-ATP using T4 polynucleotide kinase (USB, Cleveland, USA).

Fluorescence competition assays Oligonucleotides in $0.01 \mathrm{M}$ sodium phosphate $(\mathrm{pH} 7.0)$ containing $0.01 \mathrm{M} \mathrm{NaCl}$ were titrated with small, equal aliquots of concentrated 7-aminoActD solutions in the same buffer. These samples were excited at $552 \mathrm{~nm}$, and emission was measured at $613 \mathrm{~nm}$ using thermostated cells $\left(21^{\circ} \mathrm{C}\right)$ with a Perkin-Elmer LS-50 fluorescence spectrophotometer. Measurements were made in triplicate and averaged. The fluorescence of the drug that titrated into the buffer alone was subtracted to provide the fluorescence intensity change. Equilibrium constants were determined by fitting data to a singlebinding site model using a non-linear least squares algorithm that was written in MathCad (MathSoft Inc), which uses as the input, the ligand and DNA concentrations, fluorescence changes, and molar fluorescence of free 7-aminoActD.

Gel retardation and restriction endonuclease (Pvu II) protection assay Drug interactions with an 18-mer oligonucleotide were examined by a combined gel shift and Pvu II protection assay. Oligonucleotide $(1 \mu \mathrm{l}, 3.3 \mathrm{mM})$ containing a trace amount $(\leq 1 \mathrm{pM})$ that was labeled with $5{ }^{3}{ }^{32} \mathrm{P}$-phosphate was combined with $2 \mu \mathrm{l}$ of $5 \times$ sequencing buffer (200 mM Tris $\cdot \mathrm{HCl}, \mathrm{pH} 7.5,100 \mathrm{mM} \mathrm{MgCl}_{2}$, $250 \mathrm{mM} \mathrm{NaCl}$ ), $6 \mu \mathrm{l}$ of $\mathrm{H}_{2} \mathrm{O}$, and $1 \mu \mathrm{l}$ of drug solution (1.7 mM for ActD, $4.4 \mathrm{mM}$ for 2-hydroxyActD, $0.65 \mathrm{mM}$ for 7-aminoActD) or $\mathrm{H}_{2} \mathrm{O}$ (Jeong et al., 2002). The samples were incubated for $30 \mathrm{~min}$ at $4^{\circ} \mathrm{C}$, then Pvu II ( $1 \mu \mathrm{l}$, as supplied) was added and incubation was continued at $37^{\circ} \mathrm{C}$ for $30 \mathrm{~min}$. Electrophoresis was performed on a $16 \%$ non-denaturing polyacrylamide mini-gel at $4^{\circ} \mathrm{C}$ in $1 \times \mathrm{TBE}$ 


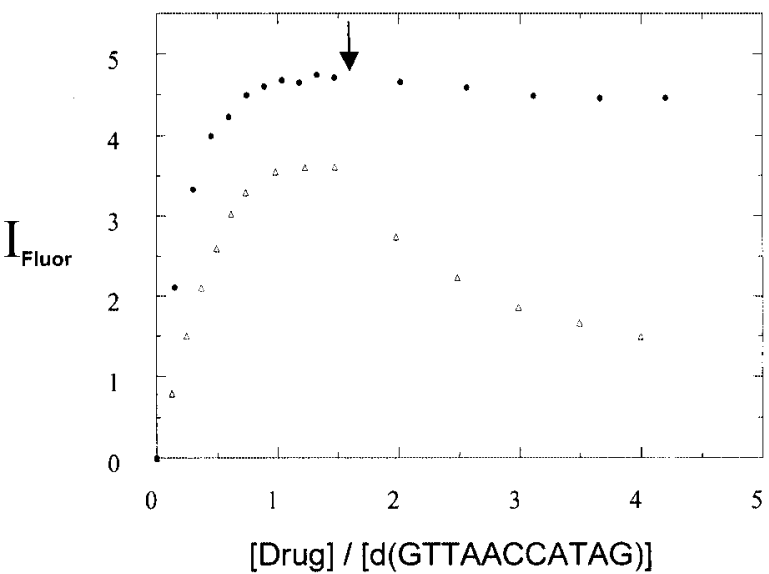

Fig. 2. Displacement of fluorescent 7-aminoActD from its complex with d(GTTAACCATAG) by competing drugs. 7AminoActD $\left(4.86 \times 10^{-5} \mathrm{M}\right)$ in $0.01 \mathrm{M}$ sodium phosphate $(\mathrm{pH}$ 7.0) containing $0.01 \mathrm{M} \mathrm{NaCl}$ was titrated into a solution of $\mathrm{d}$ (GTTAACCATAG) $\left(8.08 \times 10^{-7} \mathrm{M}\right)$ in the same buffer until saturation was nearly achieved (arrow), then a stock 2-hydroxyActD $\left(9.88 \times 10^{-5} \mathrm{M}\right)$ or ActD $\left(2.44 \times 10^{-4} \mathrm{M}\right)$ solution was added in small, equal amounts (7-amino-ActD, filled circle and open triangle to the saturation (arrow); 2-hydroxy-ActD, filled circle after arrow; ActD, open triangle after arrow). Samples were excited at $552 \mathrm{~nm}$, and emission was measured at $613 \mathrm{~nm}$. The increase in fluorescence above that expected of free 7 aminoActD is plotted. Measurements were made in triplicate and averaged.

buffer. A Pvu II protection assay was also performed with plasmid pKHQ-(+) as above with electrophoresis in $0.7 \%$ agarose.

\section{Results}

Fluorescence competition assays The fluorescence of 7aminoActD increases markedly upon binding to single- or double-stranded DNA (Sengupta and Schaer, 1978; Graves and Wadkins, 1989; Wadkins and Jovin, 1991; Wadkins et al., 1996), hence competition of an added drug for DNA can be monitored by the fluorescence intensity decrease as 7-aminoActD is displaced. Two single-stranded oligonucleotides that bind ActD with exceptionally high affinities $\left(\mathrm{K}_{\mathrm{a}}=10^{6}\right.$ to $10^{7}$ $\mathrm{M}^{-1}$ ) were examined in this assay; d(GTTAACCATAG) (Leila $\mathrm{Su}$ and Rill, unpublished), and d(CTCGACGG) referred to as PL7 by Wadkin and Jovin (1991).

As reported for other oligonucleotides (Graves and Wadkins, 1989; Wadkins and Jovin, 1991; Wadkins et al., 1996), the fluorescence of 7-aminoActD increased strongly and was blue shifted as the drug was titrated into a solution of d(GTTAACCATAG) in the absence of a competing drug (Fig. 2). A comparison of the DNA concentration with the 7aminoActD concentration near saturation is consistent with the formation of $1: 1$ complex between d(GTTAACCATAG) and 7-aminoActD with $\mathrm{K}_{\mathrm{a}}$ that is calculated to be $5.7 \times 10^{6}$ $\left(\mathrm{M}^{-1}\right.$ oligomer). Added ActD effectively competed with 7-
aminoActD, as indicated by the progressive fluorescence decrease. The fluorescence decreased to $38 \%$ of the maximum when ActD and 7-aminoActD were equimolar, a result that was consistent with the modestly higher affinity $\left(\mathrm{K}_{\mathrm{a}}=1.0\right.$ $\times 10^{7}$ ) of ActD for d(GTTAACCATAG) that was determined by titrating with ActD alone and monitoring changes in visible absorbance (Leila $\mathrm{Su}$ and Rill, unpublished). By contrast, the addition of 2-hydroxyActD to the near-saturated d(GTTAACCATAG) /7-aminoActD complex to over twofold excess caused only a $6 \%$ decrease in fluorescence, indicating that the affinity of 2-hydroxyActD is about two orders of magnitude less than that of 7-aminoActD $\left(\mathrm{K}_{\mathrm{a}}\right.$ estimated at $3 \times 10^{4} \mathrm{M}^{-1}$ from the partial isotherm). Similar results were obtained in competition assays with the second oligomer d(CTCGACGG) and with double-stranded calf thymus DNA (data not shown).

Primer extension inhibition assay Although 2-hydroxyActD showed little affinity for oligonucleotides with high affinities for ActD, other sequences might have high affinity for this modified actinomycin. Interactions of 2-hydroxyActD on ssDNA were further probed using the primer extension assay, which can detect rare, high affinity sites. Chain elongation is paused or terminated when DNA polymerase encounters a drug that is bound on the ssDNA template if the drug residence time is sufficiently long (Rill and Hecker, 1996). Locations of drug-induced primer pauses on ssDNA were identified by comparing them to dideoxy sequencing reactions.

Extension of the primer on the $(+) 1 \mathrm{~F}$ template was strongly paused by $20 \mu \mathrm{M}$ ActD or 7 -aminoActD at the same or similar sites in guanine and cytosine-rich regions on the target sequence (not shown, but see related data in Fig. 3). By contrast, $20 \mu \mathrm{M}$ 2-hydroxyActD did not pause the extension at any site in the $(+) 1 \mathrm{~F}$ target. Primer extension assays on the three other target templates produced the same results, i.e., 2hydroxyActD did not pause polymerase at the many sites of pauses that are caused by ActD, while 7-aminoActD caused pauses at essentially the same sites as ActD. Pause sites noted for the latter drugs were consistent with previous studies (Rill and Hecker, 1996).

Surprisingly, a strong and unique pause that was caused by $20 \mu \mathrm{M}$ 2-hydroxyActD was observed well downstream from the target sequence of the (+)1R template, in the LacZ gene region of the plasmid vector (Fig. 3). Pauses in this region could not have been observed in the presence of $20 \mu \mathrm{M}$ ActD or 7-aminoActD, because this drug concentration saturated the upstream binding sites. Reducing the ActD concentration to $7 \mu \mathrm{M}$ allowed the detection of a pause at the nucleotide position that was observed in the presence of $20 \mu \mathrm{M} 2$ hydroxyActD. Whereas 2-hydroxyActD caused a pause at a single nucleotide (Fig. 3A, lane 2), ActD caused pauses at several positions in the same vicinity (Fig. 3A, lane 1). Increasing the 2-hydroxyActD concentration to $100 \mu \mathrm{M}$ caused no additional pause positions (Fig. 3B), suggesting that 
(A)

(B)

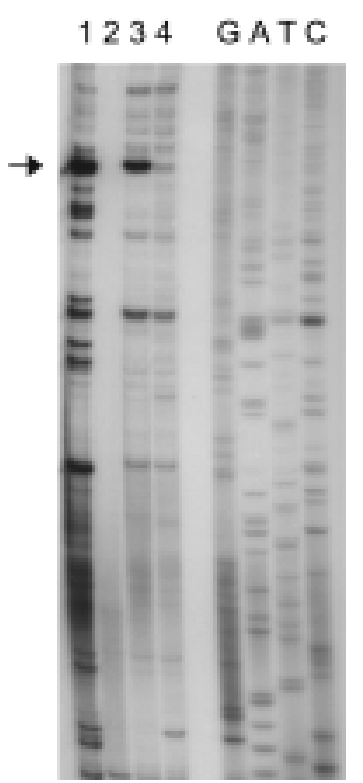

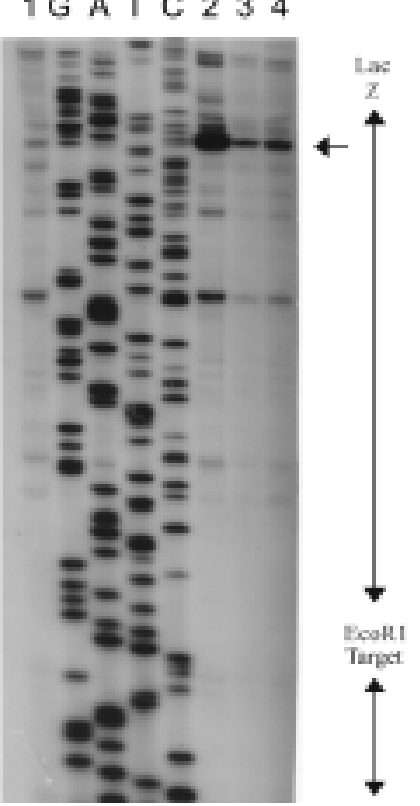

Fig. 3. Inhibition of primer extension on the single-stranded pKHQ-(+)1R template by ActD and modified ActD. Singlestranded DNA template was prepared by digesting the pKHQ$(+) 1$ vector with exonuclease after linearizing the plasmid with TfiI. (A) Primer extension by Sequenase in the presence of 7 $\mu \mathrm{M}$ ActD (lane 1), $15 \mu \mathrm{M}$ 2-hydroxyActD (lane 3), or no drug (lane 4). Lettered lanes are dideoxy sequencing reactions. (B) Concentration dependence of Sequenase pausing by 2hydroxyActD. Lane 1, control reaction (no drug). Lanes 2-4, primer extension in the presence of $100 \mu \mathrm{M}, 60 \mu \mathrm{M}$, and $30 \mu \mathrm{M}$ 2-hydroxyActD.

the two drugs bind by different sites or by different modes. This suggestion was confirmed (see below).

Since 2-hydroxyActD showed no high affinity for target sequences that were designed to minimize local secondary structures, but did bind in a LacZ sequence, it was of interest to determine whether 2-hydroxyActD can bind to other natural single-stranded DNA sequences with the potential for forming complex structures. However, the primer extension on part of phage M13 circular ssDNA (positions 6136-6254) showed no pauses in the presence of 2-hydroxyActD, although several pauses were observed for ActD.

DNA sequence and conformation around the 2hydroxyActD pause site Inspection of the ssDNA template sequence showed that 2-hydroxyActD caused Sequenase to pause at the highlighted guanine residue in a nearly perfect, 18 base pair palindrome, 5'-d(TTACGCCAGCTGGCGAAA)3'. This sequence has two additional interesting features. The central palindrome, 5'-CAGCTG-3', is the recognition sequence for restriction endonuclease Pvu II, and 5'-AGCT-3' is a well-known high affinity ActD binding site in doublestranded DNA (Chen, 1988; Rill et al., 1989; Goodisman et

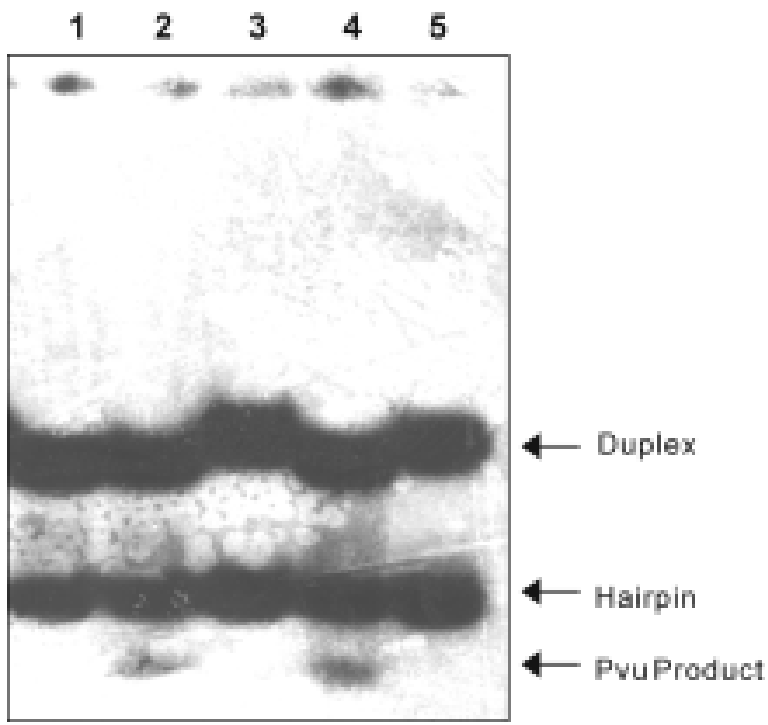

Fig. 4. Gel retardation and restriction endonuclease (PvuII) protection assay with 18-mer. The protection assay was performed using $30 \mu \mathrm{M}$ of $5{ }^{3}{ }^{32} \mathrm{P}$-end labeled 18 -mer at $4 \mu \mathrm{Ci}$. Lanes 1 and 2, 18-mer incubated without and with Pvu II. Lanes 3-5, Pvu II digestion in the presence of ActD $(170 \mu \mathrm{M})$, 2hydroxyActD $(440 \mu \mathrm{M})$, and 7-aminoActD $(65 \mu \mathrm{M})$, respectively.

al., 1992).

An 18-mer oligonucleotide with this sequence was synthesized to examine the influence of the local structure on 2-hydroxyActD binding to ssDNA. The 18-mer can form a hairpin or duplex. Equilibration between conformers was characterized by measuring the effect of oligomer concentration on electrophoretic mobility in a non-denaturing polyacrylamide gel using samples with identical amounts of 5 '-end-labeled 18-mer and increasing concentrations of unlabeled oligomer from $3 \mu \mathrm{M}$ to $300 \mu \mathrm{M}$. Two conformers were well-resolved. Below $10 \mu \mathrm{M}$, the oligomer was almost entirely in a rapidly migrating form, assigned to the hairpin. A slowly migrating form that was assigned to the duplex predominated above $60 \mu \mathrm{M}$ (not shown, but see Fig. 4).

Binding preferences of ActD, 7-aminoActD, and 2hydroxyActD for the two oligomer conformers were examined by a combined electrophoretic mobility shift and nuclease protection assay using an oligonucleotide concentration, where hairpin and duplex concentrations were nearly equal. Preferential drug binding to the duplex should increase the relative intensity of the duplex band, and reduce its mobility. Binding to the duplex should also protect the central CAGCTG site against cleavage by Pvu II, since AGCT is a high affinity ActD site (Chen, 1988; Rill et al., 1989). Conversely, preferential binding to the hairpin should increase the amount of hairpin, and have no effect on Pvu II cleavage.

A rapidly migrating band was observed after the 18-mer was incubated with restriction endonuclease Pvu II in the absence of the drug at $37^{\circ} \mathrm{C}$, demonstrating that Pvu II recognizes and cuts the palindrome in the duplex form of the 


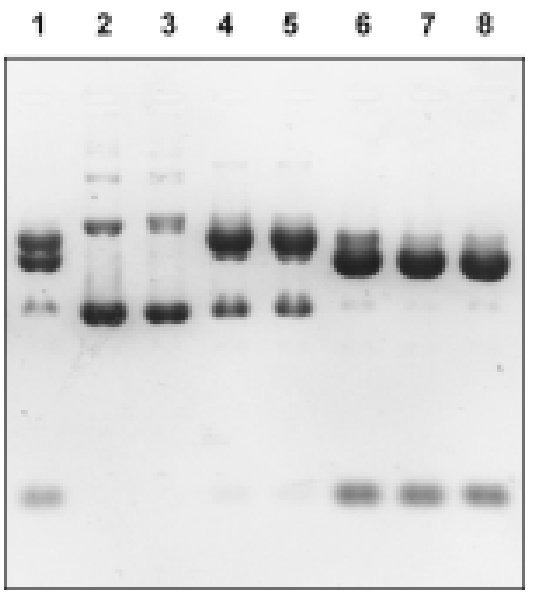

Fig. 5. Restriction endonuclase $(P v u$ II) protection assay with plasmid pKHQ-(+). Lane 1, incubation with $P v u$ II in the presence of $440 \mu \mathrm{M}$ 2-hydroxyActD; Lane 2, control digestion with no drug; Lanes 3-8, digestion in the presence of decreasing concentrations of ActD (170, 42.5, 21.3, 10.6, 5.31, and $3.40 \mu \mathrm{M}$, respectively).

18-mer (Fig. 4, lane 2). Inclusion of ActD $(170 \mu \mathrm{M})$ or 7 aminoActD $(65 \mu \mathrm{M})$ in the reaction retarded the duplex migration, and eliminated the rapidly migrating band (Fig. 4, lanes 3 and 5), indicating that both drugs bind the 5'-AGCT-3' sequence in the duplex. When the same reaction was carried out in the presence of $440 \mu \mathrm{M}$ 2-hydroxyActD, neither the duplex nor hairpin bands were retarded, and partial digestion by Pvu II was observed (Fig. 4, lane 4). The intensity of the duplex band relative to the hairpin increased when ActD was added, but 2-hydroxyActD did not affect the relative band intensities.

Mobility or concentration shifts would not be observed if dissociation of the oligomer-drug complex was rapid enough for the oligomer to migrate away from the initial zone of the drug. To eliminate this possibility, an oligomer sample containing $440 \mu \mathrm{M}$ 2-hydroxyActD was electrophoresed at $4^{\circ} \mathrm{C}$ in a gel containing uniform $100 \mu \mathrm{M}$ 2-hydroxyActD. No change in the band mobilities or relative amount of hairpin to duplex was observed (data not shown), confirming that 2hydroxyActD cannot bind with high affinity to the 18-mer hairpin.

The ability of 2-hydroxyActD to bind the 18-mer sequence in a natural dsDNA duplex was tested by comparing the rate of Pvu II cleavage of the intact plasmid in the presence of ActD or the 2-hydroxy derivative. Cleavage at the two Pvu II sites in the plasmid yielded a large and a small fragment (Fig. 5 , lane 2). Neither site was cleaved in the presence of $170 \mu \mathrm{M}$ ActD; extensive digestion, indicated by the appearance of the small fragment, was not observed until the ActD concentration was reduced to $10.6 \mu \mathrm{M}$ (Fig 5, lane 6). By contrast, at least $60 \%$ of the plasmid was cleaved at both sites in the presence of $440 \mu \mathrm{M} 2$-hydroxyActD, indicating only weak binding and protection (Fig. 5, lane 1).

\section{Discussion}

Substitution of a hydroxyl group for the amino group at the chromophore 2-position of ActD strongly decreased affinities for the normally-preferred sequences in both ssDNA and dsDNA. Currently, there is no ready explanation for the large drop in affinity for dsDNA that is caused by this substitution. High affinity dsDNA binding of ActD has been attributed to a combination of intercalation and base stacking of the chromophore, hydrogen bonding between guanines and peptide amide hydrogens and carbonyl oxygens, and reduced solvent exposure of the hydrophobic surface of the pentapeptide side chains by insertion into the minor groove (Sobell and Jain, 1972; Crothers and Muller, 1974; Krugh and Chen, 1975; Jones et al., 1988; Zhou et al., 1989, Kamitori and Takusagawa, 1992). No role was postulated for the 2amino group until the crystal structure of an actinomycin complex with dsDNA was recently reported (Kamitori and Takusagawa, 1992; Shinomiya et al., 1995). This structure suggests that hydrogen bonding interactions occur between the amino group and DNA backbone, but provides little insight into why the amino group is acceptable, yet a hydroxyl group apparently is not. It is perhaps even more perplexing that single-stranded DNA often cannot adapt to this simple structure change to afford high affinity binding, despite a much greater conformational flexibility. The atypical chromatography behavior of 2-hydroxyActD suggests that the modified drug has greater conformational variability than the parent ActD. In this case, a loss of conformational entropy on binding may account for part of the lower DNA affinity of the derivative.

Although 2-hydroxyActD showed a low overall affinity for dsDNA and ssDNA, one high affinity binding site was located within the LacZ gene. The polymerase pause occurred in a long, nearly perfect palindrome, suggesting that 2hydroxyActD might bind to a hairpin; however, an 18-mer oligonucleotide containing the palindrome did not strongly bind 2-hydroxyActD as either a hairpin or duplex. The same oligomer showed high affinity for unmodified ActD, but the AGCT site in the duplex form was preferred over the hairpin. The predicted hairpin structure of the 18-mer does not seem to represent the real conformation of the 2-hydroxyActD binding site that was detected on the long single-stranded DNA template. Presumably, a more complex structure that involves more distant sequence elements defines the binding site of 2hydroxyActD on the ssDNA template (Kang et al., 2002). The 33 nucleotide sequence, 5'CCTCTTCGCTATTACGCCAGCT G $\underline{G} C A A A G A G G-3$ ', surrounding the pause site in the original template, inclusive of the 18 nucleotide sequence previously described (in italics), has additional sequence homologies that are rich in $\mathrm{G}+\mathrm{C}$ that could create complex tertiary structures and form a hairpin with an eleven base pair stem that has one single base bulge, and a ten nucleotide loop.

Remarkably, only one strong binding site of 2hydroxyActD was found in the primer extension assays on 
single-stranded template sequences, totaling about $1000 \mathrm{nt}$ and including all possible base quartets, hence the modified actinomycin binds with a selectivity approximating that of restriction endonucleases recognizing hexanucleotide palindromes. This finding suggests that there could be an alternate route to designing drugs with high sequence selectivity and novel biological activity by targeting singlestrand DNA conformation.

Acknowledgments We thank Dr. Leila Su for guidance, and Hank Henricks of the FSU Biochemical Analysis and Synthesis Service for technical assistance.

\section{References}

Brockmann, H. (1974) History and chemistry: modification of the actinomycin molecule. Cancer Chemother. Rep. 58, 9-20.

Cantor, C. R., Warshaw, M. M. and Shapiro, H. (1970) Oligonucleotide interactions. 3. Circular dichroism studies of the conformation of deoxyoligonucleotides. Biopolymers 9, 1059-1077.

Chen, F. M. (1988) Binding specificities of actinomycin D to selfcomplementary tetranucleotide sequences -XGCY-. Biochemistry 27, 6393-6397.

Crothers, D. M. and Müller, W. (1974) Origins of base specificity in actinomycin and other DNA ligands. Cancer Chemother. Rep. 58, 97-100.

Davis, W. R., Gabbara, S., Hupe, D. and Peliska, J. A. (1998) Actinomycin D inhibition of DNA strand transfer reactions catalyzed by HIV-1 reverse transcriptase and nucleocapsid protein. Biochemistry 37, 14213-14221.

Goldberg, I. H. and Friedman, P. A. (1971) Antibiotics and nucleic acids. Annu. Rev. Biochem. 40, 775-810.

Goodisman, J., Rehfuss, R., Ward, B. and Dabrowiak, J. C. (1992) Site-specific binding constants for actinomycin D on DNA determined from footprinting studies. Biochemistry 31, 10461058.

Graves, D. E. and Wadkins, R. M. (1989) 7-Azidoactinomycin D: a novel probe for examining actinomycin D-DNA interactions. J. Biol. Chem. 264, 7262-7266.

Guo, J., Wu, T., Bes, J., Henderson, L. E. and Levin, J. G. (1998) Actinomycin D inhibits human immunodeficiency virus type 1 minus-strand transfer in in vitro and endogenous reverse transcriptase assays. J. Virol. 72, 6716-6724.

Hecker, K. H. and Rill, R. L. (1997) Synthetic polynucleotide templates for characterizing sequence-selective small molecule/ nucleic acid interactions. Anal. Biochem. 244, 67-73.

Jeeninga, R. E., Huthoff, H. T., Gultyaev, A. P. and Berkhout, B. (1998) The mechanism of actinomycin D-mediated inhibition of HIV-1 reverse transcription. Nucleic Acids Res. 26, 54725479.

Jeong, H. S., Jeong, I. C., Kim, A., Kang, S. W., Kang, H. S., Kim, Y. J., Lee, S. H. and Park, J. S. (2002) Cloning of the large subunit of replication protein A (RPA) from yeast Saccharomyces cerevisiae and its DNA binding activity through redox potential. J. Biochem. Mol. Biol. 35, 194-198.

Jones, R. L., Scott, E. V., Zon, G., Marzilli, L. G. and Wilson, W. D. (1988) An NMR investigation of the binding of the anticancer drug actinomycin $\mathrm{D}$ to oligodeoxyribonucleotides with isolated 5'd(GC)3' binding sites. Biochemistry 27, 60216026.

Kamitori, S. and Takusagawa, F. (1992) Crystal structure of the $2: 1$ complex between d(GAAGCTTC) and the anticancer drug actinomycin D. J. Mol. Biol. 225, 445-456.

Kang, J. S., Abugo, O. O. and Lakowicz, J. R. (2002) Dynamics of supercoiled and relaxed pTZ18U plasmids probed with a long-lifetime metal-ligand complex. J. Biochem. Mol. Biol. 35, 389-394.

Krugh, T. R. and Chen, Y. C. (1975) Actinomycin Ddeoxynucleotide complexes as models for the actinomycin DDNA complex. The use of nuclear magnetic resonance to determine the stoichiometry and the geometry of the complexes. Biochemistry 14, 4912-4922.

Mauger, A. B., Stuart, O. A. and Katz, E. (1991) Synthesis and properties of some peptide analogues of actinomycin D. $J$. Med. Chem. 34, 1297-1301.

Mitsuya, H., Yarchoan, R. and Broder, S. (1990) Molecular targets for AIDS therapy. Science 249, 1533-1544.

Moore, S., Kondo, M., Copeland, M. and Meienhofer, J. (1975) Synthesis and antitumor activity of 2-deamino- and N2(gamma-hydroxypropyl) actinomycin D. J. Med. Chem. 18, 1098-1101.

Phillips, D. R. and Crothers, D. M. (1986) Kinetics and sequence specificity of drug DNA interactions: an in vitro transcription assay. Biochemistry 25, 7355-7362.

Rill, R. L. and Hecker, K. H. (1996) Sequence-specific actinomycin D binding to single-stranded DNA inhibits HIV reverse transcriptase and other polymerases. Biochemistry 35, 3525-3533.

Rill, R. L., Marsch, G. A. and Graves, D. E. (1989) 7-Azidoactinomycin D: a photoaffinity probe of the sequence specificity of DNA binding by actinomycin D. J. Biomol. Struct. Dyn. 7, 591-605.

Sengupta, S. K. and Schaer, D. (1978) The interaction of 7substituted actinomycin D analogs with DNA. Biochim. Biophys. Acta 521, 89-100.

Shinomiya, M., Chu, W., Carlson, R. G., Weaver, R. F. and Takusagawa, F. (1995) Structural, physical, and biological characteristics of RNA:DNA binding agent N8 actinomycin D. Biochemistry 34, 8481-8491.

Shuman, S. (1995) Vaccinia virus DNA ligase: specificity, fidelity, and inhibition. Biochemistry 34, 16138-16147.

Sobell, H. M. (1985) Actinomycin and DNA transcription. Proc. Natl. Acad. Sci. USA 82, 5328-5331.

Sobell, H. M. and Jain, S. C. (1972) Stereochemistry of actinomycin binding to DNA. II. Detailed molecular model of actinomycin-DNA complex and its implications. J. Mol. Biol. 68, 21-34.

Takusagawa, F. (1996) Physical and biological characteristics of the antitumor drug actinomycin D analogues derivatized at $\mathrm{N}$ methyl-L-valine residues. Biochemistry 35, 13240-13249.

Takusagawa, F. (1997) Selectivity of F8-actinomycin D for RNA:DNA hybrids and its anti-leukemia activity. Bioorg. Med. Chem. 5, 1197-1207.

Tong, G. (1996) A convergent solid-phase synthesis of actinomycin analogues towards implementation of doublecombinatorial chemistry. Bioorg. Med. Chem. 4, 693-698.

Tuteja, N., Phan, T. N., Tuteja, R., Ochem, A. and Falaschi, A. 
(1997) Inhibition of DNA unwinding and ATPase activities of human DNA helicase II by chemotherapeutic agents. Biochem. Biophys. Res. Commun. 236, 636-640.

Wadkins, R. M., Jares-Erijman, E. A., Klement, R., Rudiger, A. and Jovin, T. M. (1996) Actinomycin D binding to singlestranded DNA: sequence specificity and hemi-intercalation model from fluorescence and 1H NMR spectroscopy. J. Mol. Biol. 262, 53-68.

Wadkins, R. M. and Jovin, T. M. (1991) Actinomycin D and 7aminoactinomycin $\mathrm{D}$ binding to single-stranded DNA. Biochemistry 30, 9469-9478.

Wadkins, R. M., Vladu, B. and Tung, C. S. (1998) Actinomycin $\mathrm{D}$ binds to metastable hairpins in single-stranded DNA.
Biochemistry 37, 11915-11923.

Waring, M. J. (1981) DNA modification and cancer. Annu. Rev. Biochem. 50, 159-192.

Wong-Staal, F. and Haseltine, W. A. (1992) Regulatory genes of human immunodeficiency viruses. Mol. Genet. Med. 2, 189219.

Yanisch-Perron, C., Vieira, J. and Messing, J. (1985) Improved M13 phage cloning vectors and host strains: nucleotide sequences of the M13mp18 and pUC19 vectors. Gene 33, 103119.

Zhou, N., James T. L. and Shafer, R. H. (1989) Binding of actinomycin D to [d(ATCGAT) $]_{2}$ : NMR evidence of multiple complexes. Biochemistry 28, 5231-5239. 